\title{
Colorectal carcinogenesis, colorectal cancer development and Fusobacterium nucleatum
}

\author{
Tadashi Ohara* \\ Department of Gastroenterology, Kitashinagawa Third Hospital, Kohno Clinical Medicine Research Institute, Tokyo, Japan
}

\begin{abstract}
Many studies of colorectal cancer and gut microbiota have been reported since Fusobacterium nucleatum (F. nucleatum) was reported to be involved in carcinogenesis and cancer development. The reports range from inflammation to carcinogenesis / development, avoidance from the immune system, effects on oncogenes / tumor suppressor genes, cell growth promotion, and tumor cell adhesion. There is a report suggesting that $F$. nucleatum is involved in the development of cancer, which is a poor prognosis due to large amounts of bacteria as the stage progression. In addition, F. nucleatum has been reported to be associated with CIMP-high, microsatellite instability and MLH1 hypermethylation. This is a report suggesting that $F$. nucleatum may be involved not only in the carcinogenic pathway from adenomas but also in the carcinogenic pathway from serrated adenomas. In addition, F. nucleatum is an indigenous bacterium in the oral cavity and is also a causative bacterium of periodontal disease, but there is a report that the strains of colorectal cancer and F. nucleatum in the oral cavity are the same, and its carcinogenic mechanism is drawing attention. In this review, I will outline the relationship between colorectal carcinogenesis and colorectal cancer development and $F$. nucleatum, and also address the issues.
\end{abstract}

\section{Introduction}

The first full-scale report on the relationship between colorectal cancer and gut microbiota was reported by Moore WE, et al. In 1995 [1]. They analyzed the intestinal flora of patients with colorectal polyps, those with a habit of eating Western food, and those who did not, and reported 15 species of gut microbiota as colorectal cancer risk bacteria.

Since 2010, there have been many reports on the relationship between Fusobacterium nucleatum (F. nucleatum) and colorectal cancer. Individually, among the intestinal bacteria thought to be associated with the development of colorectal cancer, we will outline F. nucleatum in particular, and how F. nucleatum is involved in carcinogenesis and cancer development. It also touches on molecular biological mechanisms and oral bacteria and describes future issues for colorectal cancer research (and prevention of colorectal cancer).

\section{Relationship between intestinal bacteria and colorectal cancer}

Although F. nucleatum is known as a periodontal disease bacterium [2], large amounts of F. nucleatum were detected in colorectal cancer specimens by the qPCR method, and its association with lymph node metastasis was also reported [3]. It has also been reported that the diversity of gut microbiota in colorectal cancer tissues was smaller than that of normal mucosal tissues distant from cancer tissues [4]. Gao Z, et al. have a high occupancy rate of Firmicutes phylum in colorectal cancer tissue, but Proteobacteria phylum is predominant in normal mucosal tissue, and at the genus level, Lactococcus and Fusobacterium occupancy rate is high in colorectal cancer tissue, and Pseudomonas and Escherichia-Shigella has been reported to have a lower occupancy rate than healthy human mucosal tissue [5]. In addition, there are two main colon carcinogenic pathways, the classical pathway and the serrated pathway, but in the former adenoma, the diversity of intestinal bacteria ( $a$-Diversity) decreased, the latter serrated lesions were not diverse, and differences in gut microbiota changes due to carcinogenic pathways have been reported [6]. Thus, colorectal cancer is strongly influenced by intestinal bacteria. In particular, F. nucleatum has been detected in colorectal cancer tissues and feces of colorectal cancer patients with reproducibility by the PCR method, and many reports have been made on its relationship with colorectal cancer.

\section{Colorectal carcinogenesis and development of colorectal} cancer due to $F$. nucleatum

It has been reported that FadA and Fap2 expressed in F. nucleatum are involved as the mechanism for colorectal carcinogenesis and colorectal cancer development in F. nucleatum. To outline these reports, FadA attaches to cells via the cell adhesion factor E-cadherin, invades cells, activates $\beta$ catenin signals, and expresses Wnt genes, transcription factors, inflammation-related genes and oncogenes. Fap2 suppresses tumor immunity by lymphocytes and overexpresses sugar chains (D-galactose- $\beta$ (1-3) -N-acetyl-D-galactosamine: NGal-GalNAc) in colorectal cancer tissues is recognized by Fap2, invades cells through it, and suppresses the expression of the tumor suppressor gene (RASA1) through the expression of miRNA21, which is involved in the development of colorectal cancer. In addition, as a report of the mechanism involved in colon carcinogenesis, TLR4 is expressed on the cell membrane and intracellular $\beta$-catenin against LPS (lipopolysaccharide), which is a bacterial cell component of $F$. nucleatum, due to F. nucleatum infection. There is a report describing the carcinogenesis cascade mediated by activation of $\beta$-catenin, in which $\beta$-catenin activity is described to occur via p21-activated kinase

*Correspondence to: Tadashi Ohara, M.D, Ph.D, Department of Gastroenterology, Kitashinagawa Third Hospital, Kohno Clinical Medicine Research Institute, 3-37 Kitashinagawa, Shinagawa, Tokyo 140-0001, Japan, Tel: +81-3-3474- 1831; Fax: +81-5461-3740; E-mail: t.ohara@kcmi.or.jp

Received: May 02, 2021; Accepted: May 12, 2021; Published: May 17, 2021 
(PAK) (TLR4 / P-PAL1 cascade) [7]. In a report describing tumor growth mediated by FadA and E-cadherin, FadA is not capable of growing normal cells without gene mutation [8]. However, it is a wellknown fact that some normal cells are accompanied by gene mutations. Furthermore, the expression of E-cadherin is also expressed in tissues other than cancer, and its expression level is not constant even in cancer and adenoma [9]. From these facts, there is a theory that disagrees with F. nucleatum of tumor growth mediated by FadA and E-cadherin. In addition, many F. nucleatum are found not only in the cancerous part of the large intestine but also in the normal colon mucosa and feces [10$11]$, and all the tissues in which F. nucleatum is found do not become cancerous. Similar to the above-mentioned FadA, E-cadherin-mediated tumor growth theory, the TLR4 / P-PAK1 cascade carcinogenesis theory is not absolute, and the details of the mechanism of F. nucleatum colon carcinogenesis have not been clarified. The above is the current situation.

On the other hand, an inflammation is deeply involved in colorectal carcinogenesis, and F. nucleatum may be involved in colorectal carcinogenesis by inducing inflammation. As a supporting report, FadA has been reported to have an inflammatory effect [12], and even though F. nucleatum infecting colorectal cancer model mice does not cause enteritis, COX-2 IL-6, and TNF- $\alpha$ is enhanced compared with the noninfected model [13]. It has also been reported that in a mouse model of colorectal cancer infected with $F$. nucleatum, tumor growth was rapid and blood cytokines were also elevated. In this report, it is stated that the microRNA that causes inflammation of the large intestine and is involved in carcinogenesis is miR21 [14]. Furthermore, it was stated that inhibition of miR21 in F. nucleatum-infected colorectal cancer model mice suppresses tumor growth, and that miR21a knockout mice are involved in cancer growth because the number and size of tumors are small and the survival time is long. In addition, the target of miR 21 is the tumor suppressor gene RASA1, and it is said that miR21 is involved in tumor growth by suppressing the expression of RASA1 [15]. It is also stated that the detected amount of $F$. nucleatum and the expression level of miR21 correlate with each other in humans.

Next, the avoidance of $F$. nucleatum infection from the immune system will be described. This is an important mechanism involved in the development of colorectal cancer. It has been reported that the number of lymphocytes in F. nucleatum-infected colorectal cancer tissue decreases according to the amount of $F$. nucleatum detected [16]. If there are many $\mathrm{T}$ cells in the cancer tissue, the $\mathrm{T}$ cells attack the cancer cells, suppress the development of the cancer, and have a positive effect on the prognosis of life. It suppresses $\mathrm{T}$ cell migration and adversely affects the prognosis of life. Although details of $\mathrm{T}$ cell migration have not been clarified, there is the report which describing the possibility of promoting apoptosis and suppressing T cell migration and Dap2 in T cells including NK cells [17]. It has been also reported that binding to the expressed TIGT (Tell immunoglobulin and ITIM domain) suppresses the cytotoxic effect of NK cells on tumors (ITIM: immunodominant tyrosine-based inhibitory motifs) [18]. Avoidance from the immune system has also been reported in mouse models of colorectal cancer [14]. The report states that infection of colorectal cancer model mice with $F$. nucleatum increases intestinal bone marrowderived immunosuppressive cells (MDSCs). MDSCs, along with regulatory $\mathrm{T}$ cells (Tregs), are cells which suppress excessive immune responses.

As mentioned above, it is thought that $F$. nucleatum infection prevents the accumulation of T cells, NK cells, dendritic cells which accumulate in the tumor to attack the tumor, and the colorectal cancer developments.

\section{F. nucleatum and colorectal cancer in feces and tissues}

Both the digital PCR method and the conventional PCR method have reported that the prognosis for life is poor in proportion to the amount of DNA in F. nucleatum in colorectal cancer tissue $[11,19]$. In Germany, Czech Republic, and Ireland, it has been reported that the prognosis of colorectal cancer is poor in cases with high DNA of F. nucleatum in feces [20] Yachida, et al. reported an increase in F. nucleatum as the depth of invasion of colorectal cancer increased. In addition, it has been reported that Atopobium paruulum and Actinomyces odontolyticus increase at the stage of carcinogenesis and decrease as they develop, which may play a role in the mechanism of carcinogenesis [21].

On the other hand, F. nucleatum in feces of patients with colorectal cancer, patients with high-grade adenoma, patients with low-grade adenoma and healthy subjects were analyzed by PCR method, and a large amount was detected in colorectal cancer patients, but the others were the same as those of healthy subjects. Therefore, some groups have reported that $F$. nucleatum may be involved in the development of colorectal cancer rather than its involvement in carcinogenesis [22]. There is also a report that it was detected in large amounts in patients with colorectal cancer and high-grade adenoma, but not significantly increased in villous adenoma and low-grade adenoma [20]. Summarizing these reports, the role of F. nucleatum in carcinogenesis may be limited. The microbial flora of feces and colon tissue is different. Large amounts of F. nucleatum in feces does not necessarily mean large amounts of F. nucleatum in tissues [20]. Considering the specificity with the disease, it is considered that the microbial flora of the large intestine mucosal tissue, which reflects the host factors, is involved in the pathology [23]. Detection of F. nucleatum in feces is relatively homeostatic and abundant in patients with colorectal cancer [24]. Because of these reasons, there is an opinion that the amount of $F$. nucleatum detected in feces should be used for the screening method for colorectal cancer, but there are many problems that must be solved in order to establish the evaluation as a biomarker in the future.

\section{Serrated adenoma / polyp (SSA / P) and F. nucleatum}

I describe the carcinogenic mechanism and gene mutation of Fusobacterium nucleatum-induced serrated adenoma / polyp (SSA/P), and the problems that need to be elucidated in the future. I have already reported on these matters [25].

The carcinogenic pathways of colorectal cancer include the classical pathway in which adenoma grows to colorectal cancer and the serrated pathway in which colorectal cancer grows from serrated adenoma. In the former adenoma, the diversity of gut flora ( $\alpha$-diversity) is reduced, but not in SSA/P, and there is a difference in the change of gut flora depending on the carcinogenic route [6]. The serrated pathway is a concept recently proposed by Torlakovic, et al. [26]. Sessile serrated adenoma / polyp (SSA/P) predominates in the right-side colon [27] and SSA/P is caused by mutations in the BRAF gene. In SSA/P, DNA methylation is observed genome-wide, and p16 and IGFBP7 are methylated to shift cells from the resting phase to the proliferative phase [28]. In addition, the methylation of the mismatch repair gene MLH1 suppresses MLH1 protein expression, causing microsatellite instability (MSI), resulting in atypia from SSA/P. Tumors caused by this atypia are called SSA/P with cytological dysplasia [29], and this concept was also proposed at WHO in 2010. The initial SSA/P lesion, which is the origin of colorectal cancer, has a flat, white-like color tone, is difficult to distinguish endoscopically from normal mucosa, and is often overlooked. Inaccurate endoscopic observations of SSA/P miss 
the detection of early-stage colorectal carcinoma [30]. The accurately diagnose SSA/P endoscopically is a future clinical issue for colorectal cancer. We have great expectations for endoscopic image analysis systems that have made remarkable progress in recent years.

F. nucleatum is known to induce gene mutations. In F. nucleatuminfected colonic mucosal tissue, CpG island methylator phenotypehigh (CIMP-high), MSI positive, and MLH1 hypermethylation are observed. In addition, there is an interesting report that SSA/P is more likely to be Fusobacterium genus-positive as the site of origin goes from the sigmoid colon to the ascending colon to the cecum to the oral-side deep colon [12]. This is not seen in classical colorectal carcinogenesis pathway. Large amounts of $F$. nucleatum have also been reported to be associated with MSI-high, MLH1 hypermethylated CIMP-high, and BRAF mutations [19]. These reports are very interesting. In addition, it has been reported that tumor immunity may be evaded in MSIhigh colorectal cancer [31]. However, not all mucosal tissues infected with $F$. nucleatum and high in MSI become cancerous and multiply, and undiscovered factors and events that cause carcinogenesis may be hidden, and details of carcinogenesis are still unclear. We would like to pay attention to future research on the details of gene mutations caused by F. nucleatum, SSA/P carcinogenesis and BRAF gene mutations.

\section{Oral bacteria and colorectal cancer}

It has been reported that the same strain of F. nucleatum was detected in $40 \%$ or more of patients by comparing the saliva of patients with colorectal cancer and the strains of colorectal cancer tissue by arbitrarily primed PCR [32]. This report indicates that F. nucleatum in colorectal cancer may be derived from F. nucleatum in the oral cavity. However, by the time saliva F. nucleatum reaches the large intestine, it must pass through the stomach in an acidic environment and the small intestine, which is rich in bile acids. This raises the question of whether F. nucleatum really survives through the stomach and small intestine until it reaches the large intestine. There are reports that answer this question. It is reported that when $F$. nucleatum was intravenously injected into a mouse model of colorectal cancer, a large amount of F. nucleatum was detected in colorectal cancer tissue [33]. This report shows that F. nucleatum migrates to hematogenously and colonizes colon tissue. Furthermore, it has been reported that bloodstream disease occurs in the pathophysiology of periodontal disease in which F. nucleatum increases [34]. From the above reports, it is considered that oral $F$. nucleatum may reach the large intestine tissue through the bloodstream. However, the mechanism of how a trace amount of $F$. nucleatum in the blood proliferates after reaching the large intestine tissue has not been clarified.

\section{F. nucleatum and autophagy}

Normally, it is known that when stress is applied to cells, autophagy is promoted, and apoptosis escapes due to survival. F. nucleatumpositive cases are often found in cases of colorectal cancer recurrence, chemotherapy resistance, and short time to recurrence. The reason for this is that some groups have reported that $F$. nucleatum may promote autophagy and suppress apoptosis [35]. They report in vitro that the autophagy-promoting effect of $F$. nucleatum surpasses apoptosis. There is an interesting report describing the autophagy of F. nucleatum.

\section{Conclusions and future tasks}

I have summarized and outlined the research articles on the relationship between F. nucleatum, colorectal carcinogenesis and colorectal cancer development which have been reported so far.
The pathophysiology of $F$. nucleatum and colorectal cancer is very complex. Many related research articles have been reported, some of which are negative about the involvement of $F$. nucleatum in colorectal carcinogenesis. The involvement of $F$. nucleatum in colorectal carcinogenesis remains unsolved and requires further analysis. On the other hand, the report on the involvement of F. nucleatum in the progression of colorectal cancer is convincing. The representative are reports on immune avoidance. It is unclear whether the immune evasive action of F. nucleatum is due to F. nucleatum itself or synergistically with gene mutations such as MSI, but it greatly contributes to the development of colorectal cancer. In addition, suppression of apoptosis by F. nucleatum autophagy is also a factor involved in the development of colorectal cancer. Elucidation of the more detailed mechanism for the relationship between F. nucleatum and the development of colorectal cancer is awaited.

F. nucleatum has been reported to be involved not only in colorectal cancer but also in esophageal cancer and pancreatic cancer [36]. We hope that the pathophysiology of F. nucleatum infection will be elucidated with the advent of new findings in the future.

\section{Disclosure of COI}

There is no COI to disclose.

\section{References}

1. Moore WE, Moore LH (1995) Intestinal floras of populations that have a high risk of colon cancer. Appl Environ Microbial 61: 3202-3207.

2. Han YW, Fardini Y, Chen C, Iacampo KG, Peraino VA, et al. (2010) Term stillbirth caused by oral Fusobacterium nucleatum. Obsset Gynecol 115: 442-445.

3. Castellarin M, Warren RL, Freeman JD, Dreolini L, Krzywinsky M (2012) Fusobacterium nucleatum infection is prevent in human colorectal carcinoma. Genome Res 22: 299-306.

4. Chen W, Liu F, Ling Z, Tong X, Xiang C (2012) Human intestinal lumen and mucosaassociated microbiota in patients with colorectal cancer. PLoS One 7: e39743.

5. Gao Z, Guo B, Gao R, Zhu Q, Qin H (2015) Microbiota dysbiosis is associated with colorectal cancer. Front Microbial 6: 20.

6. Peters BA, Dominianni C, Shaprio JA, Church TR, Wu J (2016) The gut microbiota in conventional and serrated precursors of colorectal cancer. Microbiome 4: 69.

7. Chen Y, Peng Y, Yu J, Chen T, Wu Y (2017) Inversive Fusobacterium nucleatum activates beta-catenin signaling in colorectal cancer via a TLR4/P-PARK1 cascade. Oncotarget 8: 31802-31814.

8. Gholizadeh P, Eslami H, Kafil HS (2017) Carcinogenesis mechanisms of Fusobacterium nucleatum. Biomed Pharmacother 89: 918-925.

9. Jungck M, Grünhage F, Spengler U, Dernac A, Mathiak M (2004) E-cadherin expression is homogeneously reduced in adenoma from patients with familial adenomatous polyposis: an immunohistochemical study of E-cadherin, beta-catenin and cyclooxygenase-2 expression. Int J Colorectal Dis 19: 438-445. [Crossref]

10. Yamaoka Y, Suehiro Y, Hashimoto S, Hoshida K, Fujimoto M (2018) Fusobacterium nucleatum as a prognostic marker of colorectal cancer in a Japanese population. $J$ Gastroenterol 53: 517-524.

11. Tahara T, Yamamoto E, Suzuki H, Maruyama R, Chung W (2014) Fusobacterium in colonic flora and molecular features of colorectal carcinoma. Cancer Res 74: 13111318.

12. Rubinstein MR, Wang X, Liu W, Hao Y, Cai G (2013) Carcinogenesis by modulating E- cadherin/ $\beta$-catenin signaling via its FadA adhesin. Cell Host Microbe 14: 195-206.

13. Kostic AD, Chun E, Robertson L, Glickman JN, Gallini CA, Michaud M (2013) Fusobacterium nucleatum potentiates intestinal tumorigenesis and modulates the tumor-immune microenvironment. Cell Host Microbe 14: 207-215.

14. Shi C, Yang Y, Xia Y, Okugawa Y, Yang J (2016) Novel evidence for an oncogenic role of microRNA-21 in colitis-associates colorectal cancer. GUT 65: 1470-1481. 
15. Yang Y, Weng W, Peng J, Hong L, Yang L (2017) Fusobacterium nucleatum increases proliferation of colorectal cancer cells and tumor development in mice by activating Toll-like receptor 4 signaling to Nuclear Factor- $\mathrm{\kappa B}$, and up regulating expression of microRNA-21. Gastroenterol 152: 851-866.

16. Mima K, Sukawa Y, Nishihara R, Quian ZR, Yamauchi M (2015) Fusobacterium nucleatum and T cells in colorectal carcinoma. JAMA Oncol 1: 653-661.

17. Gur C, Ibrahim Y, Isaacson B, Yamin R, Abed J (2015) Binding of the Fap2 protein of Fusobacterium nucleatum to human inhibitory receptor TIGIT protects tumors from immune cell attach. Immunity 42: 344-355.

18. Mima K, Nishihara R, Qian ZR, Cao Y, Sukawa Y (2016) Fusobacterium nucleatum in colorectal carcinoma tissue and patient prognosis. GUT 65: 1973-1980.

19. Flanagan L, Schmid J, Ebert M, Soucek P, Kunicka T (2014) Fusobacterium nucleatum associates with stages of colorectal neoplasia development, colorectal cancer and disease outcome. Eur J Clin Microbiol Infect Dis 33: 1381-1390.

20. Yachida S, Mizutani S, Shiroma H, Shiba S, Nakajima T (2019) Metagenomic and metabolomic analyses reveal distinct stage-specific phenotypes of the gut microbiota in colorectal cancer. Nat Med 25: 968-976.

21. Amity EL, Werner S, Vital M, Pieper DH, Hofler D (2017) Fusobacterium and colorectal cancer: causal factor or passenger? Results from a large colorectal cancer screening study. Carcinogenesis 38: 781-788.

22. Zoetendal EG, von Wright A, Vilpponen-Salmela T, Ben-Amor K, Akkermans AD (2002) Mucosa-associated bacteria in the human gastrointestinal tract are uniformly distributed along the colon and differ from the community recovered from feces. Appl Environ Microbiol 68: 3401-3407.

23. Hashemi Goradel N, Heidarzadeh S, Jahangiri S, Farhood B, Mortezaee K (2019) Fusobacterium nucleatum and colorectal cancer: A mechanistic overview. $J$ Cell Physiol 234: 2337-2344.

24. Ohara T (2021) Sessile serrated adenoma/polyps (SSA/P) carcinogenic mechanism and gene mutations caused by Fusobacterium nucleatum -Problems to be clarified in the future.
25. Torlakovic E, Snover DC (1996) Serrated adenomatous polyposis in humans. Gastroenterol 110: 748-755.

26. Ban S, Mitomi H, Horiguchi H, Sato H, Shimizu M (2014) Adenocarcinoma arising in sessile serrated adenoma/ polyp (SSA/P) of the colon: clinicopathological study of eight lesions. Pathol Int 64: 123-133.

27. Kaji E, Uraoka T, Kato J, Hiraoka S, Suzuki H (2012) Externalization of saw-tooth architecture in small serrated polyps implies the presence of methylation of IGFBP7. Dig Dis Sci 57: 1261-1270.

28. Matthew FK (2013) Sessile serrated polyps: an important route to colorectal cancer. $J$ Natl Compr Cnnc Netw 11: 1586-1594.

29. Rashtak S, Rego R, Sweetser SR, Sinicrope FA (2017) Sessile serried polyps and colon cancer prevention. Cancer Prev Res 10: 270-278. [Crossref]

30. Hamada T, Zhang X, Mima K, Bullman S, Sukawa Y (2018) Fusobacterium nucleatum in colorectal cancer relate to immune response differentially by tumor microsatellite instability status. Cancer Immunol Res 6: 1327-1336.

31. Komiya Y, Shimomura Y, Higurashi T, Sugi Y, Arimoto J (2019) Patients with colorecta cancer have identical strains of Fusobacterium nucleatum in their colorectal cancer and oral cavity. GUT 68: 1335-1337.

32. Abed J, Emgard JE, Zamir G, Faroja M, Almogy G (2016) Fusobacterium nucleatum colorectal adenocarcinoma enrichment by binding to tumor-expressed Gal-GalNAc. Cell Host Microbe 20: 215-225.

33. Ashare A, Stanford C, Hancock P, Stark D, Lilli K (2009) Chronic liver disease impairs bacterial clearance in a human model of induced bacteremia. Clin Transl Sci 2: 199205.

34. Yu T, Guo F, Yu Y, SunT, Ma D (2017) Fusobacterium nucleatum promotes chemoresistance ton colorectal cancer by modulating autophagy. Cell 170: 548-563.

35. Yamamura K, Baba Y, Nakagawa S, Mima K, Miyake K (2016) Human microbiome Fusobacterium nucleatum in esophageal cancer tissue is associated with prognosis. Clin Cancer Res 22: 5574-5581. [Crossref]

36. Fan X, Alekseyenko AV, Wu J, Peters BA (2018) Human oral microbiome and prospective risk for pancreatic cancer: a population-based nested case-control study. GUT 67: 120-127.

Copyright: (C2021 Ohara T. This is an open-access article distributed under the terms of the Creative Commons Attribution License, which permits unrestricted use, distribution, and reproduction in any medium, provided the original author and source are credited. 